\title{
Lymphatic spread is related to VEGF-C expression and D2-40-positive myofibroblasts in intrahepatic cholangiocarcinoma
}

\author{
Shinichi Aishima ${ }^{1,2}$, Yunosuke Nishihara ${ }^{2}$, Tomohiro Iguchi ${ }^{2}$, Kenichi Taguchi ${ }^{3}$, \\ Akinobu Taketomi ${ }^{4}$, Yoshihiko Maehara ${ }^{4}$ and Masazumi Tsuneyoshi ${ }^{2}$ \\ ${ }^{1}$ Department of Pathology, Hamanomachi Hospital, Fukuoka, Japan; ${ }^{2}$ Department of Anatomic Pathology, \\ Graduate School of Medical Sciences, Kyushu University, Fukuoka, Japan; ${ }^{3}$ Laboratory of Cancer Pathology, \\ Institute for Clinical Research, National Kyushu Cancer Center Fukuoka, Fukuoka, Japan and ${ }^{4}$ Department of \\ Surgery and Science, Graduate School of Medical Sciences, Kyushu University, Fukuoka, Japan
}

\begin{abstract}
Lymph node metastasis via lymphatic vessels is related with an adverse outcome in many tumors. It is unclear whether lymphatic spread needs the development of the new lymphatic vessels or the expression of lymphangiogenetic factor in intrahepatic cholangiocarcinoma. The aim of this study was to assess the role of lymphangiogenesis, vascular endothelial growth factor-C (VEGF-C) expression, and D2-40-positive myofibroblastic cells for lymphatic spread and patient outcome in 88 cases of intrahepatic cholangiocarcinoma. We also assessed VEGF-C expression in 15 cases of metastatic lymph nodes. There was a significant correlation between lower lymphatic vessel density in the tumor center and positive lymphatic invasion $(P=0.0100)$. Poorly differentiated cholangiocarcinoma showed higher lymphatic vessel density in the tumor periphery and in the peritumoral area $(P=0.0315$ and $P=0.0360$, respectively). Lymphatic invasion was observed higher in the peritumoral area $(63 \%, 24 / 38)$ and in the tumor periphery $(79 \%, 30 / 38)$ than in the tumor center $(27 \%$, $9 /$ 38). There was no significant correlation between the proliferative lymphatic vessels and pathologic features; however, Iymphatic invasion was significantly associated with VEGF-C expression $(P=0.0006)$, and the VEGF-C expression was seen in 12 of 15 cases $(80 \%)$ of metastatic lymph node. Nodal metastasis was correlated with D2-40-positive myofibroblasts $(P=0.0161)$. VEGF-C expression was an independent prognostic factor by multivariate survival analysis $(P=0.0131)$. Our findings suggest that VEGF-C has an important role in lymphatic invasion via the preexisting lymphatic vessels in the tumor margin, and that lymphangiogenesis does not play a direct role in lymphatic metastasis. D2-40-positive myofibroblasts may contribute to lymphatic metastasis. Modern Pathology (2008) 21, 256-264; doi:10.1038/modpathol.3800985; published online 11 January 2008
\end{abstract}

Keywords: VEGF-C; D2-40; lymphatic vessel density; cholangiocarcinoma

Lymph node involvement is the first step of tumor metastasis in most human cancers and serves as a prognostic indicator. The lymphatic vasculature is important as the major routes for metastatic spread to the lymph node. In the last decade, significant progress has been made in the understanding of lymphangiogenesis and cancer metastasis. ${ }^{1,2}$ Several types of human cancer can induce the formation of lymphatic vessels, ${ }^{3-6}$ whereas other cancers do not actively induce lymphangiogenesis and simply invade existing lymphatic vessels. ${ }^{7-13}$ A positive

Correspondence: Dr S Aishima, MD, Department of Pathology, Hamanomachi Hospital, 3-5-27 Maizuru, Chuo-ku, Fukuoka 810-8539, Japan.

E-mail: aishima-s@hamanomachi.jp

Received 23 July 2007; revised 29 October 2007; accepted 31 October 2007; published online 11 January 2008 relationship between the intratumoral or peritumoral lymphatic vessel density and lymphatic spreads has been reported in head and neck squamous cell carcinoma, ${ }^{3,14,15}$ cutaneous melanoma, ${ }^{16,17}$ lung cancer, ${ }^{18}$ and pancreatic endocrine tumor. ${ }^{19,20}$

Vascular endothelial growth factor-C (VEGF-C) has been found to be the common lymphangiogenetic factor, acting predominantly via VEGF receptor-3 (VEGFR-3), which is expressed by lymphatic endothelial cells, ${ }^{21}$ and VEGF-C strongly promoted the growth of tumor-associated lymphatic vessels. ${ }^{22-26}$ Some clinical studies have shown a correlation between tumor VEGF-C expression and lymph node metastasis. ${ }^{27-29}$

Intrahepatic cholangiocarcinoma remains an unfortunate liver tumor with a high mortality rate, because many patients diagnosed with cholangiocarcinoma cannot be offered curative treatment. ${ }^{30}$ 
One of the major hallmark of cholangiocarcinoma is its lymph node metastasis. The lymphatic invasion and lymph node involvement are important prognostic indicators for intrahepatic cholangiocarcinoma; ${ }^{31-34}$ however, lymph node metastasis and lymphangiogenesis at the mechanistic level in intrahepatic cholangiocarcinoma are poorly understood.

The recently developed monoclonal antibody, D2-40, recognizes a mucin-like transmembrane glycoprotein, podoplanin, which is expressed in lymphatic endothelial cells. ${ }^{35}$ Besides being reactive with lymphatic endothelium, D2-40 staining has also been observed in normal tissue, such as myofibroblasts and myoepithelial cells. In addition, podoplanin was also expressed in several tumors, including squamous cell carcinoma, germ cell tumor, and mesothelioma, raising a possibility that it may have biologic functions in tumor cells. ${ }^{35-37}$ Thus, immunohistochemical examination of D2-40-positive non-lymphatic cells was explored to understand tumor progression.

The aim of this study was to determine intratumoral or peritumoral lymphatic vessel density by immunostaining for D2-40, proliferative lymphatic vessel as a lymphangiogenesis by double staining for D2-40/Ki67, VEGF-C expression, and intratumoral D2-40-positive myofibroblasts in intrahepatic cholangiocarcinoma, which predominantly showed a mass-forming shape and correlated with pathologic data and patient survival.

\section{Materials and methods}

\section{Patients and Materials}

Immunohistochemical examination was performed on 88 cases of primary intrahepatic cholangiocarcinoma specimens and 15 cases of lymph nodes with metastatic cancer cells obtained between the years 1986 and 2005. The lymph nodes were collected during surgery on the primary tumor. They were retrieved from the files of the Department of Pathology of the Kyushu University. The clinicopathological characteristics of the resected primary intrahepatic cholangiocarcinomas are summarized in Table 1. On the basis of the definition by the Japanese Liver Cancer Study Group, ${ }^{38}$ we examined 88 cases of mass-forming type of intrahepatic cholangiocarcinoma. The cases of intraductal growth type and periductal infiltrating type were excluded because neither of the tumor type was suited for the assessment of intratumoral lymphatic vessel density. The mean age was 64 years (range $=33-90$ ); the group included 57 men and 31 women. Of these, 27 had metastatic spread to the resected lymph nodes. All patients had surgery and some postoperative chemotherapy. Sections that contained areas of adenocarcinoma with adjacent non-cancerous tissue were selected. The studies
Table 1 Summary of clinicopathologic features of ICC

\begin{tabular}{lc}
\hline Age, mean \pm s.d. (range) (years) & $64 \pm 11(33-90)$ \\
Gender, M/F & $57 / 31$ \\
Tumor size, mean \pm s.d. (range) (cm) & $4.4 \pm 2.2(1.0-12.0)$ \\
Histologic differentiation & 21 \\
Well & 39 \\
Moderately & 28 \\
Poorly & \\
Lymphatic invasion & 38 \\
Present & 50 \\
Absent & \\
Nodal metastasis & \\
Present & 27 \\
Absent & 61 \\
Survival & \\
Alive & 36 \\
Died of disease & 48 \\
Died of other disease & 4 \\
\hline
\end{tabular}

were conducted with ethical approval of the Kyushu University Human Ethics Committee.

\section{Immunohistochemistry and Evaluation}

Four-micrometer thick sections were cut and deparaffinized through xylene and ethanol. Afterwards, the endogenous peroxidase activity was blocked by methanol containing $0.3 \%$ hydrogen peroxidase. Antigen retrieval was performed in a microwave oven (citrate buffer, $\mathrm{pH}$ 6.0) for D2-40, MIB-1, and $\alpha$-smooth muscle actin. The sections for VEGF-C were digested with $0.1 \%$ trypsin solution at $37^{\circ} \mathrm{C}$ for $30 \mathrm{~min}$. The sections were incubated overnight at $4^{\circ} \mathrm{C}$ with the primary antibodies against podoplanin (mouse monoclonal, D2-40; Nichirei Bioscience), VEGF-C (goat polyclonal, N-19, 1:200; Santa Cruz Biotechnology), Ki67 (mouse monoclonal, MIB-1, 1:100, Immunotech, Marseille, France) and $\alpha$-smooth muscle actin (mouse monoclonal, 1:400; Sigma, St Louis, MO, USA). The sections were then incubated with the appropriate biotinylated secondary antibodies and streptavidin-biotin-peroxidase complex (Nichirei Pharmaceutical, Tokyo, Japan). Peroxidase reactivity was visualized using 3,3'-diaminobenzidine. Known breast cancer tissue was used as a positive control for VEGF-C staining ${ }^{39}$ and normal lymph node was used as a positive control for D2-40 staining. Immunoreactivity for VEGF-C was considered positive when the cytoplasmic staining was observed in more than $10 \%$ of cancer cells. Spindle-shaped stromal cells reactive for both anti- $\alpha$-smooth muscle actin and D2-40 in the tumor-associated stroma were regarded as D2-40-positive myofibroblasts. Two observers (SA and $\mathrm{YN}$ ) evaluated and interpreted the results of immunohistochemical staining without the knowledge of the clinical data of each patient. 
Assessment of Lymphatic Vessel Density and Lymphatic Invasion

Quantitative analysis of lymphatic vessel density was performed in sections that were single-stained for D2-40. The most vascularized areas within tumor ('hot spots') were chosen at low magnification $(\times 40$ or $\times 100)$ and vessels were counted at a representative high magnification $\left(\times 400 ; 0.152 \mathrm{~mm}^{2} ; 0.44 \mathrm{~mm}\right.$ diameter). The lymphatic vessels were counted in three fields and were recorded as total number per unit area. Three different regions were assessed separately for each section by the hot spot method. They were as follows: (a) tumor center, (b) tumor periphery-a 2-mm wide band of tumor immediately adjacent to the invasive front, and (c) peritumoral non-cancerous area-a 2-mm wide band of host tissue adjacent to the invasive front (Figure 1). Single endothelial cells were counted as individual microvessels. Endothelial staining in large vessels with tunica media and non-endothelial structures were disregarded in lymphatic vessel counts. Lymphatic invasion was considered evident if at least one tumor cell cluster was clearly visible inside the D2-40-stained vascular space. The presence of lymphatic invasion was evaluated in the three areas (tumor center, tumor periphery, and peritumoral area).

\section{Double Staining for D2-40/Ki67}

The ongoing tumor lymphangiogenesis would be the analysis of proliferating lymphatic endothelial cells, which can be assessed by a double immunostaining with D2-40 to stain lymphatic vessels, together with Ki67 to stain proliferative cells. Sixty slides demonstrating high lymphatic vessel density were selected for double staining. Ki67 staining was developed with peroxidase-conjugated secondary antibody, and then D2-40 immunostaining was developed with alkaline phosphatase-conjugated secondary

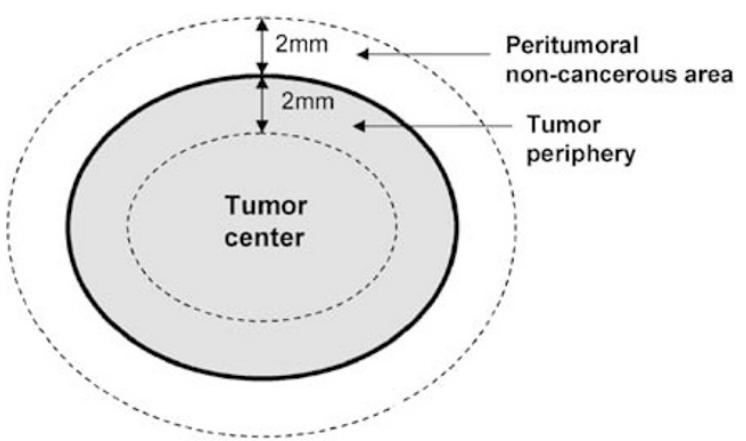

Figure 1 Three areas examined for lymphatic vessel density and for lymphatic invasion. The peritumoral area is defined as a $2-\mathrm{mm}$ wide band of host tissue adjacent to the invasive front. The tumor periphery is defined as a 2-mm wide band of tumor immediately adjacent to the invasive front, and tumor center is defined as an inner center area. Tumor is shown in gray. antibody. The rate of double-labeled vessels was determined by counting the nuclei of tumorassociated D2-40-positive vessels (100 nuclei in each tumor).

\section{Statistical Analysis}

Mean values of lymphatic vessel density and proliferative lymphatic vessels were compared using the Student's $t$-test. A statistical analysis to compare the relationships between the VEGF-C expression, the D2-40-stromal cells, and the clinicopathological parameters was made using the $\chi^{2}$-test. Patients' survival was taken as the period of survival between surgery and the date of the last follow-up or death due to disease. The data from patients who died of causes unrelated to the disease were censored at the time of death. The survival curves were plotted using the Kaplan-Meier method and compared using the log-rank test. We conducted a univariate analysis of the prognostic factors with the $\chi^{2}$-test and multivariate analysis with Cox's regression model. $P<0.05$ was considered statistically significant.

\section{Results}

Lymphatic Vessel Density, Lymphatic Invasion, and Proliferative Lymphatic Vessels

In the non-cancerous liver tissue, a few D2-40positive lymphatic vessels arranged in linear pattern were seen in the portal tracts. Peritumoral D2-40positive lymphatic vessels in the area adjacent to the tumor margin had open lumina around the blood vessels (Figure 2a). Intratumoral lymphatic vessels were mainly detected in the tumor periphery. Lymphatic vessels in the tumor periphery tended to have a small open lumen and to be associated with entrapped portal tracts, but D2-40-positive lymphatic vessels in the tumor center often showed narrow lumen or collapsed (Figure 2b). The mean lymphatic vessel density was as follows: 9.98 (s.d. =8.79, range $=0-33$ ) in the tumor center, 18.28 (s.d. $=7.81$, range $=2-38$ ) in the tumor periphery, and 19.60 (s.d. $=5.61$, range $=6-34$ ) in the peritumoral area.

Cancer cell invasion to the D2-40-positive lymphatic vessels was seen in 38 cases (43\%). Of the 38 cases, lymphatic invasion was observed in 24 cases $(63 \%)$ in the peritumoral area, 30 cases $(79 \%)$ in the tumor periphery, and 9 cases (24\%) in the tumor center. D2-40-positive vessels containing cancer invasion were often dilated in the peritumoral area or in the tumor periphery (Figure 2c).

Proliferative lymphatic vessels were determined in the tumor periphery and in the tumor center separately. The rate of Ki67-positive lymphatic vessel nuclei was $1.2 \%(\mathrm{~s} . \mathrm{d} .=1.15)$ in the tumor periphery and $0.9 \%$ (s.d. $=0.98$ ) in the tumor center 

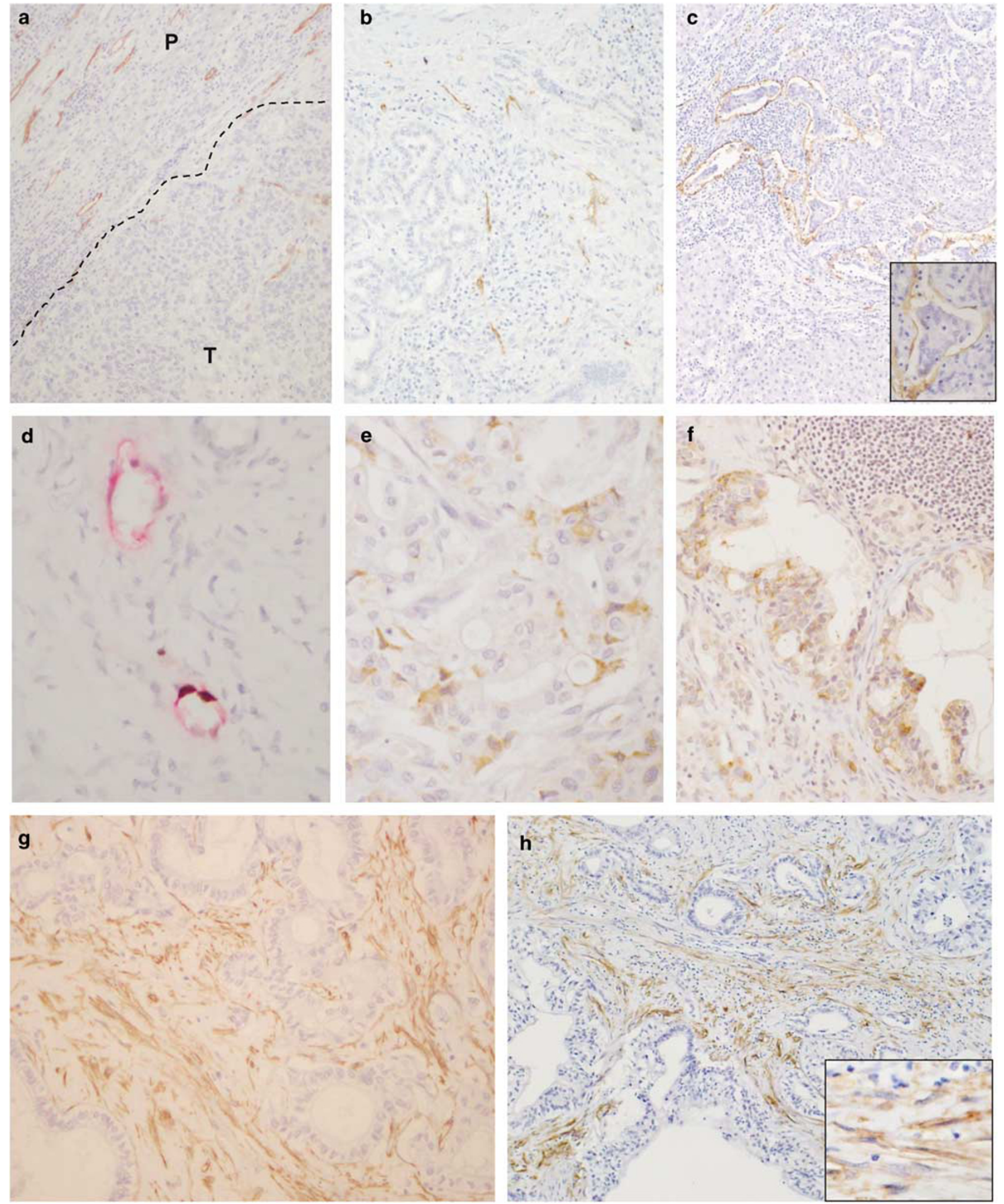

Figure 2 (a) D2-40-positive lymphatic vessels are present both in the peritumoral areas (P) and in the tumor periphery (T). Dotted lines indicate invasive front. (b) D2-40-positive vessels in the tumor center are often collapsed or narrow lumen. (c) Several clusters of cancer cells are present in the dilated vessels with D2-40-positive at the peritumoral areas. (d) Ki67-positive lymphatic vessel nuclei (arrow) were detected by double staining for D2-40/Ki67. (e) VEGF-C staining was seen in the cytoplasm of cancer cells in primary tissue. (f) Cancer cell nest proliferating in lymph node is positive for VEGF-C. (g) Most tumors with stromal desmoplasia contain $\alpha$-smooth muscle actin-positive stromal cells with myofibroblastic differentiation. (h) $\alpha$-Smooth muscle actin-positive myofibroblastic cells are often composed of D2-40-positve spindle-shaped cells (inset), indicative of D2-40-positive myofibroblasts. 
(Figure 2d). In the peritumoral areas, proliferating lymphatic vessels were found in only four cases.

\section{VEGF-C in Primary Tumor and in Lymph Nodes, and} D2-40-Positive Myofibroblasts

Positive staining for VEGF-C has heterogeneous distribution within tumor and was observed in cancer cells in 26 cases $(30 \%)$. Cancer cells showed granular cytoplasmic staining (Figure 2e) and VEGF$\mathrm{C}$ was also detected at low levels in fibroblastic cells and in blood vessel endothelial cells. By comparison, $12(80 \%)$ of 15 cases of the metastatic lymph nodes showed the cytoplasmic staining for VEGF-C in cancer nests (Figure 2f).

Intratumoral fibrous stroma contains spindleshaped myofibroblastic cells with $\alpha$-smooth muscle actin- and D2-40-positive staining in 50 cases (57\%) (Figure 2g and $\mathrm{h}$ ).

\section{Correlation of Pathologic Parameters}

As shown in Table 2, the proportion of lymphatic vessel density in the peritumoral area and tumor periphery was higher in the cases with poorly differentiated histology than in the cases with well and moderately differentiated histology $(P=0.0315$ and $P=0.0360$, respectively). The lower lymphatic vessel density in the tumor center was significantly associated with the presence of lymphatic invasion $(P=0.0100)$. There was no significant correlation between the proliferative rate of lymphatic vessels and pathologic features. Positive VEGF-C expression of cancer cells was significantly correlated with the presence of lymphatic invasion $(P=0.0006)$; however, it did not correlated with nodal metastasis. There was no correlation between VEGF-C expression and lymphatic vessel density in any region. The rate of $\mathrm{D} 2-40$-positive myofibroblasts was significantly higher in the cases with nodal metastasis than in the cases without nodal metastasis $(P=0.0161)$ (Table 3).

\section{Outcome after Surgery and Prognostic Factors}

Univariate survival analysis showed that VEGF-C expression, tumor size, histologic differentiation, lymphatic invasion, and nodal metastasis were prognostic indicators. Intratumoral or peritumoral lymphatic vessel density had no influence on patient outcome. In addition, VEGF-C expression, histologic differentiation, and nodal metastasis were independent prognostic factors according to multivariate analysis (Figure 3 and Table 4).

\section{Discussion}

Several studies have shown that peritumoral lymphatic vessels are associated with lymph node metastasis, ${ }^{8,14,17,18,27,40,41}$ whereas other studies, only for head and neck squamous cell carcinoma, showed that intratumoral lymphatic vessels, and not peritumoral lymphatic vessel density, are important for lymphatic spreads. ${ }^{3,14,15}$ Thus, we first examined the

Table 2 Correlation of lymphatic vessel density with pathologic features

\begin{tabular}{|c|c|c|c|c|c|c|c|}
\hline & \multirow[t]{2}{*}{ Cases } & \multicolumn{6}{|c|}{ Lymphatic vessel density } \\
\hline & & $\begin{array}{l}\text { Tumor center } \\
\text { (mean } \pm \text { s.d.) }\end{array}$ & $\mathrm{P}$-value & $\begin{array}{l}\text { Tumor periphery } \\
\text { (mean } \pm \text { s.d.) }\end{array}$ & $\mathrm{P}$-value & $\begin{array}{l}\text { Peritumoral area } \\
\text { (mean } \pm \text { s.d.) }\end{array}$ & $\mathrm{P}$-value \\
\hline \multicolumn{8}{|l|}{ Tumor size } \\
\hline$\leq 4.0 \mathrm{~cm}$ & 47 & $10.60 \pm 9.20$ & 0.4829 & $19.45 \pm 6.82$ & 0.1358 & $19.92 \pm 4.65$ & 0.5783 \\
\hline$>4.0 \mathrm{~cm}$ & 41 & $9.27 \pm 8.36$ & & $16.95 \pm 8.71$ & & $19.24 \pm 6.57$ & \\
\hline \multicolumn{8}{|l|}{ Histology } \\
\hline $\begin{array}{l}\text { Well/ } \\
\text { moderately }\end{array}$ & 60 & $9.62 \pm 9.23$ & 0.5762 & $17.07 \pm 7.32$ & $0.0315^{*}$ & $18.75 \pm 4.94$ & $0.0360^{*}$ \\
\hline Poor & 28 & $10.75 \pm 7.87$ & & $20.89 \pm 8.32$ & & $21.43 \pm 6.55$ & \\
\hline \multicolumn{8}{|c|}{ Lymphatic invasion } \\
\hline Present & 38 & $7.24 \pm 7.21$ & $0.0100^{*}$ & $19.53 \pm 8.39$ & 0.1952 & $19.47 \pm 5.86$ & 0.8524 \\
\hline Absent & 50 & $12.06 \pm 9.37$ & & $17.34 \pm 7.29$ & & $19.70 \pm 5.46$ & \\
\hline \multicolumn{8}{|c|}{ Nodal metastasis } \\
\hline Present & 27 & $8.26 \pm 8.07$ & 0.2246 & $18.11 \pm 8.37$ & 0.8910 & $18.19 \pm 5.87$ & 0.1151 \\
\hline Absent & 61 & $10.74 \pm 9.05$ & & $18.36 \pm 7.63$ & & $20.23 \pm 5.41$ & \\
\hline \multicolumn{8}{|c|}{ VEGF-C expression } \\
\hline Positive & 26 & $7.19 \pm 5.748$ & 0.0537 & $20.19 \pm 8.30$ & 0.1388 & $20.35 \pm 4.69$ & 0.4233 \\
\hline Negative & 62 & $11.15 \pm 9.59$ & & $17.48 \pm 7.52$ & & $19.29 \pm 5.95$ & \\
\hline
\end{tabular}

*Indicates $P<0.05$. 
Table 3 Correlation of VEGF-C expression and D2-40-positive stromal cells with pathologic features

\begin{tabular}{|c|c|c|c|c|c|}
\hline & \multirow[t]{2}{*}{ Cases } & \multicolumn{2}{|c|}{ VEGF-C protein } & \multicolumn{2}{|c|}{ D2-40-positive stromal cells } \\
\hline & & Positive (\%) & $\mathrm{P}$-value & Positive (\%) & $\mathrm{P}$-value \\
\hline \multicolumn{6}{|l|}{ Tumor size } \\
\hline$\leq 4.0 \mathrm{~cm}$ & 47 & $15(32 \%)$ & 0.7738 & $26(55 \%)$ & 0.7612 \\
\hline$>4.0 \mathrm{~cm}$ & 41 & $11(27 \%)$ & & $24(59 \%)$ & \\
\hline \multicolumn{6}{|l|}{ Histology } \\
\hline Well/moderately & 60 & $17(65 \%)$ & 0.9092 & $32(53 \%)$ & 0.4623 \\
\hline Poor & 28 & $9(32 \%)$ & & $18(64 \%)$ & \\
\hline \multicolumn{6}{|l|}{ Lymphatic invasion } \\
\hline Present & 38 & $19(50 \%)$ & $0.0006^{*}$ & $25(66 \%)$ & 0.2063 \\
\hline Absent & 50 & $7(14 \%)$ & & $25(50 \%)$ & \\
\hline \multicolumn{6}{|l|}{ Nodal metastasis } \\
\hline Present & 27 & $12(44 \%)$ & 0.0743 & $21(78 \%)$ & 0.0161 * \\
\hline Absent & 61 & $14(23 \%)$ & & $29(48 \%)$ & \\
\hline
\end{tabular}

*Indicates $P<0.05$.
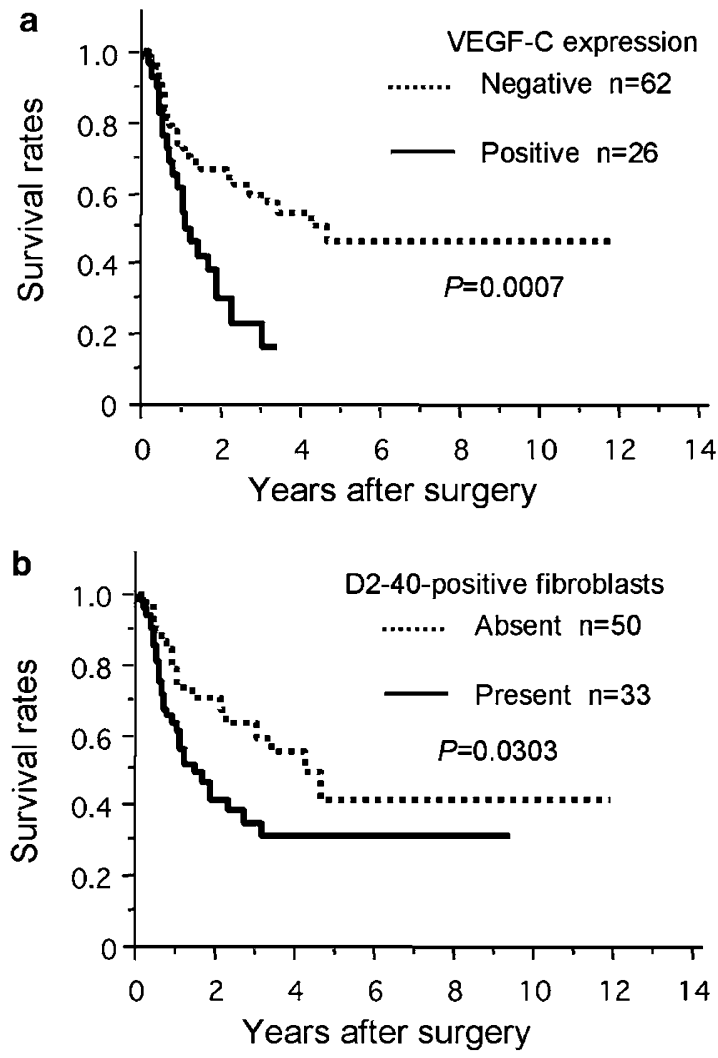

Figure 3 (a) The survival rates of patients with positive VEGF-C expression were worse than those of patients in negative VEGF-C expression $(P=0.0007)$. (b) The survival rates of patients with D2-40-positive stromal cells were worse than those of patients without D2-40-positive stromal cells $(P=0.0303)$.

intratumoral or peritumoral lymphatic vessel density in intrahepatic cholangiocarcinomas, and detected the following: (1) lymphatic vessel density was much lower in the tumor center than in the tumor periphery; (2) lymphatic vessels in the tumor center were frequently collapsed, whereas peritumoral lymphatic vessels showed open lumen; and (3) lower lymphatic vessel density in the tumor center was significantly correlated with the presence of lymphatic invasion. It has been reported that pancreatic cancer and melanoma demonstrated collapsed intratumoral lymphatic vessels and dilated peritumoral lymphatic vessels. ${ }^{7,12}$ Some studies have shown an inverse correlation between tumor aggressiveness and intratumoral lymphatic vessel density.,4,10,11 These results suggest that intratumoral lymphatic vessels, especially in the tumor center, are destructed by the tumor growth and the lymphatic vessel is preserved in the tumor border in intrahepatic cholangiocarcinoma. In the current study, tumor invasion into the lymphatic vessels was frequently detected in the tumor periphery or in the peritumoral area. Therefore, lymphatic vessels at the tumor boundary may offer an opportunity for nodal metastasis in intrahepatic cholangiocarcinoma.

Although a correlation of lymphatic vessel density with histologic differentiation has not been reported in any cancer type, our study demonstrated that poorly differentiated types showed higher lymphatic vessel density in the tumor periphery and in the peritumoral area. Poorly differentiated cholangiocarcinoma sometimes grows an infiltrative fashion between a preexisting structure without disturbing the tissue architecture, such as vascular or lymphatic structure. This finding may account for the description that infiltrative breast carcinomas had many lymphatic vessels compared with expansive type. ${ }^{42}$

There is considerable debate whether cancer cells can metastasize by invasion of preexisting lymphatic vessels or by the formation and invasion of new lymphatic vessels within tumors. Although lymphatic vessels with proliferating nuclei have been reported in some cancers ${ }^{3,4,39,43}$ our result by 
Table 4 Univariate and stepwise multivariate survival analysis for ICC

\begin{tabular}{|c|c|c|c|c|c|}
\hline & \multirow[t]{2}{*}{ No. of cases } & \multicolumn{2}{|c|}{ Univariate analysis } & \multicolumn{2}{|c|}{ Stepwise multivariate analysis } \\
\hline & & 5-year survival & P-value & P-value & Hazard (95\% CI) ratio \\
\hline LVD in the tumor center & & & 0.7367 & & \\
\hline High & 44 & $38 \%$ & & & \\
\hline Low & 44 & $35 \%$ & & & \\
\hline LVD in the tumor periphery & & & 0.1790 & & \\
\hline High & 43 & $29 \%$ & & & \\
\hline Low & 45 & $43 \%$ & & & \\
\hline LVD in the peritumoral area & & & 0.4135 & & \\
\hline High & 43 & $24 \%$ & & & \\
\hline Low & 45 & $46 \%$ & & & \\
\hline VEGF-C expression & & & 0.0007 & 0.0131 & \\
\hline Negative & 62 & $49 \%$ & & & 1 \\
\hline Positive & 26 & $0 \%$ & & & $2.146[0.222,0.846]$ \\
\hline D2-40-positive stromal cells & & & 0.0303 & & \\
\hline Present & 50 & $25 \%$ & & & \\
\hline Absent & 38 & $51 \%$ & & & \\
\hline Tumor size & & & 0.0186 & & \\
\hline$\leq 40 \mathrm{~mm}$ & 47 & $44 \%$ & & & \\
\hline$>40 \mathrm{~mm}$ & 41 & $26 \%$ & & & \\
\hline Histologic differentiation & & & 0.0004 & 0.0020 & \\
\hline Well/moderately & 60 & $49 \%$ & & & 1 \\
\hline Poorly & 28 & $7 \%$ & & & $2.542[1.138,4.003]$ \\
\hline Lymphatic invasion & & & $<0.0001$ & & \\
\hline Present & 38 & $16 \%$ & & & \\
\hline Absent & 50 & $55 \%$ & & & \\
\hline Nodal metastasis & & & $<0.0001$ & $<0.0001$ & \\
\hline Present & 27 & $0 \%$ & & & $4.330[1.562,8.391]$ \\
\hline Absent & 61 & $50 \%$ & & & 1 \\
\hline
\end{tabular}

double staining of D2-40/Ki67 revealed the low rate of proliferating lymphatic vessels in intrahepatic cholangiocarcinoma. The result indicates that most intratumoral lymphatic vessels are preexisting vessels and lymphangiogenesis indeed weakly occurs in intrahepatic cholangiocarcinomas, and that preexisting lymphatic vessels, rather than newly formed ones, tend to be targets of invasion by cancer cells. The hypothesis is in line with the finding that a large number of lymphatic invasions were observed in the peritumoral tissue, which rarely has proliferative lymphatic vessels. Like our result, lymphangiogenesis do not always occur in breast cancers. ${ }^{11,44}$ It was demonstrated that increased lymphangiogenic activity is not required for the lymphatic spread in pancreatic ductal adenocarcinoma, ${ }^{7,9}$ and prostatic adenocarcinoma. ${ }^{8,22}$ Several studies showed that increased lymphatic perimeters and areas were found to be correlated with presence of lymphatic metastasis. ${ }^{17,39,40,42}$ Therefore, lymphatic invasion in intrahepatic cholangiocarcinoma is independent of the active lymphangiogenesis, but other possible factors are expected for the lymphatic spread. Indeed, the incidence of lymph node metastasis increased in proportion to an increase in the expression of matrix metalloproteinase-9 in intrahepatic cholangiocarcinoma. ${ }^{45}$ The stromal desmoplasia is significantly related to the lymphatic invasion. $^{46}$

Nodal metastasis has been promoted in tumors overexpressing VEGF-C. ${ }^{23}$ A recent study showed that VEGF-C expression of cancer cells was correlated with lymph node metastasis in intrahepatic cholangiocarcinoma. ${ }^{47}$ In our study, VEGF-C expression in cancer cells is significantly correlated with lymphatic invasion and clinical outcome, and this expression in the metastatic lymph nodes was observed in 12 of 15 cases (80\%). A few studies showed that VEGF-C support the entry of cancer cells into peritumoral lymphatic vessels. ${ }^{7,27}$ These results suggest that VEGF-C plays a role in the formation of lymph node metastasis without active lymphangiogenesis.

D2-40 reactivity is also observed in myofibroblasts of the prostate, and myoepithelial cells of breast 
glands and of salivary glands. ${ }^{35}$ These myofibroblasts and myoepithelial cells are closely related to the biologic functions of the ductal epithelial cells. Podoplanin, which is recognized by D2-40 antibody, was also expressed in several tumors and might have a role in tumor migration or invasion. ${ }^{36,37}$ In our study, D2-40-positive spindle-shaped cells with myofibroblastic feature diffusely proliferated in the tumor with nodal metastasis. Although it became clear that cancer cells respond to fibroblasts differentially, depending on the type of fibroblasts and epithelial cells, ${ }^{48}$ fibroblasts isolated from cancer tissue induced invasion of proliferation of cancer cells. ${ }^{49,50}$ Thus, cancer-associated fibroblasts contribute to cancer growth and metastatic progression, and are expected to the drug targets for anticancer therapy. ${ }^{51}$ Podoplanin has not previously been linked to tumor-associated stroma; however, intratumoral D2-40-positive myofibroblasts may play a role in tumor progression due to the close interaction of cancer cells and D2-40-positive myofibroblasts.

These findings suggest that VEGF-C has an important role in lymphatic invasion, and that the preexisting lymphatic vessels in the tumor margin are sufficient for lymphatic metastasis. Lymphangiogenesis might occur in intrahepatic cholangiocarcinoma, but it does not play a direct role in lymphatic metastasis. D2-40-positive myofibroblasts may contribute to tumor metastasis with a tumor-stroma interaction.

\section{References}

1 Stacker SA, Achen MG, Jussila L, et al. Lymphangiogenesis and cancer metastasis. Nat Rev Cancer 2002;2:573-583.

2 Nathanson SD. Insights into the mechanisms of lymph node metastasis. Cancer 2003;98:413-423.

3 Beasley NJ, Prevo R, Banerji S, et al. Intratumoral lymphangiogenesis and lymph node metastasis in head and neck cancer. Cancer Res 2002;62:1315-1320.

4 Straume O, Jackson DG, Akslen LA. Independent prognostic impact of lymphatic vessel density and presence of low-grade lymphangiogenesis in cutaneous melanoma. Clin Cancer Res 2003;9:250-256.

5 Van der Auwera I, Van Laere SJ, Van den Eynden GG, et al. Increased angiogenesis and lymphangiogenesis in inflammatory versus noninflammatory breast cancer by real-time reverse transcriptase-PCR gene expression quantification. Clin Cancer Res 2004;10:7965-7971.

$6 \mathrm{He} \mathrm{Y}$, Rajantie I, Ilmonen M, et al. Preexisting lymphatic endothelium but not endothelial progenitor cells are essential for tumor lymphangiogenesis and lymphatic metastasis. Cancer Res 2004;64:3737-3740.

7 Schneider M, Buchler P, Giese N, et al. Role of lymphangiogenesis and lymphangiogenic factors during pancreatic cancer progression and lymphatic spread. Int J Oncol 2006;28:883-890.

8 Roma AA, Magi-Galluzzi C, Kral MA, et al. Peritumoral lymphatic invasion is associated with regional lymph node metastases in prostate adenocarcinoma. Mod Pathol 2006;19:392-398.

9 Sipos B, Kojima M, Tiemann K, et al. Lymphatic spread of ductal pancreatic adenocarcinoma is independent of lymphangiogenesis. J Pathol 2005;207:301-312.

10 Vleugel MM, Bos R, van der Groep P, et al. Lack of lymphangiogenesis during breast carcinogenesis. J Clin Pathol 2004;57:746-751.

11 Williams CS, Leek RD, Robson AM, et al. Absence of lymphangiogenesis and intratumoural lymph vessels in human metastatic breast cancer. J Pathol 2003;200:195-206.

12 Padera TP, Kadambi A, di Tomaso E, et al. Lymphatic metastasis in the absence of functional intratumor lymphatics. Science 2002;296:1883-1886.

13 Leu AJ, Berk DA, Lymboussaki A, et al. Absence of functional lymphatics within a murine sarcoma: a molecular and functional evaluation. Cancer Res 2000;60:4324-4327.

14 Kyzas PA, Geleff S, Batistatou A, et al. Evidence for lymphangiogenesis and its prognostic implications in head and neck squamous cell carcinoma. J Pathol 2005;206:170-177.

15 Maula SM, Luukkaa M, Grenman R, et al. Intratumoral lymphatics are essential for the metastatic spread and prognosis in squamous cell carcinomas of the head and neck region. Cancer Res 2003;63:1920-1926.

16 Dadras SS, Lange-Asschenfeldt B, Velasco P, et al. Tumor lymphangiogenesis predicts melanoma metastasis to sentinel lymph nodes. Mod Pathol 2005;18:1232-1242.

17 Massi D, Puig S, Franchi A, et al. Tumour lymphangiogenesis is a possible predictor of sentinel lymph node status in cutaneous melanoma: a case-control study. J Clin Pathol 2006;59:166-173.

18 Renyi-Vamos F, Tovari J, Fillinger J, et al. Lymphangiogenesis correlates with lymph node metastasis, prognosis, and angiogenic phenotype in human nonsmall cell lung cancer. Clin Cancer Res 2005;11: 7344-7353.

19 Rubbia-Brandt L, Terris B, Giostra E, et al. Lymphatic vessel density and vascular endothelial growth factor-C expression correlate with malignant behavior in human pancreatic endocrine tumors. Clin Cancer Res 2004;10:6919-6928.

20 Sipos B, Klapper W, Kruse ML, et al. Expression of lymphangiogenic factors and evidence of intratumoral lymphangiogenesis in pancreatic endocrine tumors. Am J Pathol 2004;165:1187-1197.

21 He Y, Rajantie I, Pajusola K, et al. Vascular endothelial cell growth factor receptor 3-mediated activation of lymphatic endothelium is crucial for tumor cell entry and spread via lymphatic vessels. Cancer Res 2005;65:4739-4746.

22 Wong SY, Haack H, Crowley D, et al. Tumor-secreted vascular endothelial growth factor-C is necessary for prostate cancer lymphangiogenesis, but lymphangiogenesis is unnecessary for lymph node metastasis. Cancer Res 2005;65:9789-9798.

23 Chen Z, Varney ML, Backora MW, et al. Downregulation of vascular endothelial cell growth factor$\mathrm{C}$ expression using small interfering RNA vectors in mammary tumors inhibits tumor lymphangiogenesis and spontaneous metastasis and enhances survival. Cancer Res 2005;65:9004-9011.

24 Karpanen T, Egeblad M, Karkkainen MJ, et al. Vascular endothelial growth factor C promotes tumor 
lymphangiogenesis and intralymphatic tumor growth. Cancer Res 2001;61:1786-1790.

25 Mandriota SJ, Jussila L, Jeltsch M, et al. Vascular endothelial growth factor-C-mediated lymphangiogenesis promotes tumour metastasis. EMBO J 2001;20: 672-682.

26 Skobe M, Hawighorst T, Jackson DG, et al. Induction of tumor lymphangiogenesis by VEGF-C promotes breast cancer metastasis. Nat Med 2001;7:192-198.

27 Gombos Z, Xu X, Chu CS, et al. Peritumoral lymphatic vessel density and vascular endothelial growth factor $\mathrm{C}$ expression in early-stage squamous cell carcinoma of the uterine cervix. Clin Cancer Res 2005;11: 8364-8371.

28 Ueda M, Hung YC, Terai Y, et al. Vascular endothelial growth factor- $\mathrm{C}$ expression and invasive phenotype in ovarian carcinomas. Clin Cancer Res 2005;11:3225-3232.

29 Choi WW, Lewis MM, Lawson D, et al. Angiogenic and lymphangiogenic microvessel density in breast carcinoma: correlation with clinicopathologic parameters and VEGF-family gene expression. Mod Pathol 2005;18:143-152.

30 Khan SA, Thomas HC, Davidson BR, et al. Cholangiocarcinoma. Lancet 2005;366:1303-1314.

31 Inoue K, Makuuchi M, Takayama T, et al. Long-term survival and prognostic factors in the surgical treatment of mass-forming type cholangiocarcinoma. Surgery 2000;127:498-505.

32 Aishima SI, Taguchi KI, Sugimachi K, et al. c-erbB-2 and C-Met expression relates to cholangiocarcinogenesis and progression of intrahepatic cholangiocarcinoma. Histopathology 2002;40:269-278.

33 Kitagawa Y, Nagino M, Kamiya J, et al. Lymph node metastasis from hilar cholangiocarcinoma: audit of 110 patients who underwent regional and paraaortic node dissection. Ann Surg 2001;233:385-392.

34 Kawarada Y, Yamagiwa K, Das BC. Analysis of the relationships between clinicopathologic factors and survival time in intrahepatic cholangiocarcinoma. Am J Surg 2002;183:679-685.

35 Schacht V, Dadras SS, Johnson LA, et al. Up-regulation of the lymphatic marker podoplanin, a mucin-type transmembrane glycoprotein, in human squamous cell carcinomas and germ cell tumors. Am J Pathol 2005;166:913-921.

36 Yuan P, Temam S, El-Naggar A, et al. Overexpression of podoplanin in oral cancer and its association with poor clinical outcome. Cancer 2006;107:563-569.

37 Wicki A, Christofori G. The potential role of podoplanin in tumour invasion. Br J Cancer 2007;96:1-5.

38 Liver Cancer Study Group of Japan. The General Rules for the Clinical and Pathological Study of Primary
Liver Cancer, 4th edn. Kanahara Publication: Tokyo, 2000.

39 Kinoshita J, Kitamura K, Kabashima A, et al. Clinical significance of vascular endothelial growth factor-C (VEGF-C) in breast cancer. Breast Cancer Res Treat 2001;66:159-164.

40 Dadras SS, Paul T, Bertoncini J, et al. Tumor lymphangiogenesis: a novel prognostic indicator for cutaneous melanoma metastasis and survival. Am J Pathol 2003;162:1951-1960.

41 Franchi A, Gallo O, Massi D, et al. Tumor lymphangiogenesis in head and neck squamous cell carcinoma: a morphometric study with clinical correlations. Cancer 2004;101:973-978.

42 Van der Auwera I, Colpaert C, Van Marck E, et al. Lymphangiogenesis in breast cancer. Am J Surg Pathol 2006;30:1055-1056.

43 Van der Auwera I, Van den Eynden GG, Colpaert CG, et al. Tumor lymphangiogenesis in inflammatory breast carcinoma: a histomorphometric study. Clin Cancer Res 2005;11:7637-7642.

44 Agarwal B, Saxena R, Morimiya A, et al. Lymphangiogenesis does not occur in breast cancer. Am J Surg Pathol 2005;29:1449-1455.

45 Shirabe K, Shimada M, Kajiyama K, et al. Expression of matrix metalloproteinase-9 in surgically resected intrahepatic cholangiocarcinoma. Surgery 1999;126: 842-846.

46 Kajiyama K, Maeda T, Takenaka K, et al. The significance of stromal desmoplasia in intrahepatic cholangiocarcinoma: a special reference of 'scirrhoustype' and 'nonscirrhous-type' growth. Am J Surg Pathol 1999;23:892-902.

47 Park BK, Paik YH, Park JY, et al. The clinicopathologic significance of the expression of vascular endothelial growth factor-C in intrahepatic cholangiocarcinoma. Am J Clin Oncol 2006;29:138-142.

48 Maehara N, Matsumoto K, Kuba K, et al. NK4, a four-kringle antagonist of HGF, inhibits spreading and invasion of human pancreatic cancer cells. Br J Cancer 2001;84:864-873.

49 Olumi AF, Grossfeld GD, Hayward SW, et al. Carcinoma-associated fibroblasts direct tumor progression of initiated human prostatic epithelium. Cancer Res 1999;59:5002-5011.

50 Sato N, Maehara N, Goggins M. Gene expression profiling of tumor-stromal interactions between pancreatic cancer cells and stromal fibroblasts. Cancer Res 2004;64:6950-6956.

51 Micke P, Ostman A. Exploring the tumour environment: cancer-associated fibroblasts as targets in cancer therapy. Expert Opin Ther Targets 2005;9:1217-1233. 